\title{
Different angioregulatory activity of monovalent galectin-9 isoforms
}

\author{
Ed Aanhane $^{1} \cdot$ Iris A. Schulkens ${ }^{1,2} \cdot$ Roy Heusschen ${ }^{1,3} \cdot$ Kitty Castricum $^{2} \cdot$ Hakon Leffler $^{4} \cdot$ Arjan W. Griffioen $^{1}$. \\ Victor L. Thijssen ${ }^{1,2}$ (1)
}

Received: 26 June 2017 / Accepted: 26 February 2018 / Published online: 2 March 2018

(c) The Author(s) 2018. This article is an open access publication. This article is an open access publication

\begin{abstract}
Galectin-9 consists of two peptide-linked carbohydrate recognition domains (CRDs), but alternative splicing and proteolytic processing can give rise to multiple galectin-9 isoforms. Some of these consist of a single CRD and can exert different functions in cell biology. Here, we explored the role of these galectin-9 isoforms in endothelial cell function and angiogenesis. For this, we compared the effects of the two separate CRDs (Gal-9N and Gal-9C) with the tandem repeat galectin-9M on endothelial cell proliferation, migration, sprouting and tube formation in vitro as well as on angiogenesis in vivo using the chicken chorioallantoic membrane (CAM) assay. Galectin-9 isoforms significantly affected proliferation in quiescent endothelial cells and migration in activated endothelial cells. Interestingly, both monovalent gal-9 CRDs displayed opposite effects compared to gal-9M on proliferation and migration. Sprouting was significantly inhibited by gal-9C, while all isoforms appeared to stimulate tube formation. Angiogenesis in vivo was hampered by all three isoforms with predominant effects on vessel length. In general, the isoforms induced only subtle concentration-dependent effects in vitro as well as in vivo. Collectively, the effects of different galectin-9 isoforms in endothelial cell biology depend on the cellular activation status. While opposing effects can be observed on a cellular level in vitro, all galectin-9 isoforms hamper angiogenesis in vivo. This warrants further investigation of the regulatory mechanisms that underlie the diverging roles of galectin- 9 isoforms in endothelial cell biology since this could provide therapeutic opportunities.
\end{abstract}

Keywords Angiogenesis · Blood vessels $\cdot$ Galectin $\cdot$ Sprouting $\cdot$ Tube formation $\cdot$ Cancer

Electronic supplementary material The online version of this article (https://doi.org/10.1007/s10456-018-9607-8) contains supplementary material, which is available to authorized users.

Ed Aanhane and Iris A. Schulkens have contributed equally to this work.

Victor L. Thijssen

v.thijssen@vumc.nl

1 Angiogenesis Laboratory, Department of Medical Oncology, Cancer Center Amsterdam, VU University Medical Center, De Boelelaan 1118, 1081 HV Amsterdam, The Netherlands

2 Angiogenesis Laboratory, Department of Radiation Oncology, Cancer Center Amsterdam, VU University Medical Center, De Boelelaan 1118, 1081 HV Amsterdam, The Netherlands

3 Present Address: Laboratory of Hematology, GIGA-Research, University of Liège, Liege, Belgium

4 Section Microbiology, Immunology, Glycobiology, Institute of Laboratory Medicine, Lund University, Lund, Sweden

\section{Introduction}

Galectins are glycan-binding proteins that interact with specific carbohydrate ligands on a variety of substrates, including extracellular matrix proteins, cell surface glycoproteins and glycolipids [1]. Alterations in galectin expression are frequently associated with cancer [2], and members of the galectin family have been linked to different key processes of tumor progression [3, 4]. For example, galectins are involved in cellular processes like proliferation and apoptosis, thereby contributing to tumor cell transformation [5, 6]. In addition, galectins have been shown to promote tumor progression by facilitating tumor immune escape, tumor metastasis and induction of tumor angiogenesis [3, 4, 7]. Regarding the latter, we and others have shown that endothelial cells require galectin-1 for proper function during tumor angiogenesis and that tumor cells can induce angiogenesis by secretion of galectin-1 [8-11]. Likewise, angiostimulatory activity has been ascribed to galectin-3 [12-14] and galectin-8 [15]. 
All this identifies galectins as important regulators of tumor angiogenesis.

In an effort to identify the key angioregulatory galectins, we have previously performed extensive galectin expression profiling in endothelial cells of different origin [16]. Besides confirming the expression of galectin- 1 , galectin-3, and galectin-8, we also observed endothelial galectin- 9 expression [16]. Galectin-9 consists of two carbohydrate recognition domains (CRD) that are covalently connected by a short linker peptide $[1,17]$. The length of this linker, and hence the flexibility and valency of the protein, is regulated by posttranscriptional splicing of two exons, i.e., exon 5 and 6 [18]. Consequently, three bivalent galectin- 9 isoforms are frequently reported in literature, i.e., gal-9L (or gal-9FL; full length galectin-9), gal-9M (or gal-9 $\Delta 5$; lacks exon 5) and gal-9S (or gal-9 $\Delta 5 / 6$; lacks exons 5 and 6) [17]. Our investigations into the role of galectin-9 in endothelial cell biology and angiogenesis resulted in the identification of two additional transcript variants that, apart from exons 5 and 6 , also lack exon 10, i.e. gal-9M $\Delta 10$ and gal-9S $\Delta 10$ $[16,19]$. Interestingly, splicing of exon 10 results in a frame shift and a premature stop codon, thereby giving rise to a truncated galectin-9 protein that lacks the C-terminal CRD [19]. While it remains to be confirmed whether such variants exist as stable proteins in vivo, it has been shown that the linker peptide of certain gal-9 isoforms is susceptible to proteolytic digestion [20]. This results in separation of both galectin-9 CRDs, which might serve as a mechanism to inactivate the chemoattractant activity of gal-9 [20]. In addition, the individual domains have been shown to exert distinct effects on T cells [21]. In the current study, we explored whether separation of the galectin-9 CRDs affects the role of galectin- 9 on endothelial cell function and angiogenesis.

\section{Materials and methods}

\section{Cell culture}

Primary human umbilical vein endothelial cells (HUVECs) were isolated as described previously [16]. Cells were cultured in $0.2 \%$ gelatin-coated culture flasks at $37{ }^{\circ} \mathrm{C} / 5 \% \mathrm{CO} 2$ in RPMI (Gibco) containing 10\% fetal calf serum (Invitrogen) and $10 \%$ human serum supplemented with L-glutamine (Invitrogen) and penicillin/streptomycin (Invitrogen). At confluency, cells were passaged 1:3 for a maximum of 3 passages.

\section{Recombinant galectin-9 protein production and isolation}

Competent BL21(DE3) cells (New England Biolabs) were transformed with the appropriate plasmid (pGEX-G9NT/
pGEX-G9CT; a kind gift of Dr. Nozomu Nishi, Life Sciences Research Centre, Kagawa University, Kagawa, Japan) and grown on LB-agar plates containing $100 \mu \mathrm{g} /$ $\mathrm{mL}$ ampicillin according standard protocols. A single colony was used to inoculate $100 \mathrm{~mL}$ LB medium containing $100 \mu \mathrm{g} / \mathrm{mL}$ ampicillin and grown overnight at $30{ }^{\circ} \mathrm{C}$. The overnight culture was diluted in $1.6 \mathrm{~L} \mathrm{~TB}$ medium (1.2\% Tryptone, $2.4 \%$ Yeast extract, $0.4 \%$ glycerol, $72 \mathrm{mM} \mathrm{K} 2 \mathrm{HPO} 4,17 \mathrm{mM} \mathrm{KH} 2 \mathrm{PO} 4)$ containing $100 \mu \mathrm{g} /$ $\mathrm{mL}$ ampicillin an grown to OD 0.4-0.6. Cultures were cooled on ice, induced with $1 \mathrm{mM}$ IPTG and grown overnight at $22{ }^{\circ} \mathrm{C}$. The next day, pellets were harvested by centrifugation, weighed and dissolved in $4 \mathrm{~mL} / \mathrm{g}$ of fresh, chilled B-PER solution (Thermo Fisher Scientific) containing $1 \times$ Halt protease cocktail (Thermo Fisher Scientific) $1 \mathrm{mM}$ PMSF (Sigma Aldrich), $2 \mathrm{mM}$ EDTA, $4 \mathrm{mM}$ $\beta$-mercaptoethanol, and 2.73 units/ $\mu \mathrm{L}$ DNaseI (Qiagen). The solution was incubated at room temperature for $15^{\prime}$ and centrifuged at $4500 \mathrm{rpm}$. The supernatant was loaded onto a $\beta$-Lactosyl Sepharose column (prepared as previously described by [22]. Prior to supernatant loading, the column was washed with $300 \mathrm{~mL}$ washing solution $(1 \times$ PBS containing $0.02 \% \mathrm{NaN} 3,4 \mathrm{mM} \beta$-mercaptoethanol and $2 \mathrm{mM}$ EDTA) and blocked with $300 \mathrm{~mL}$ blocking solution (3\% BSA in $1 \times$ PBS). Following overnight incubation at $4{ }^{\circ} \mathrm{C}$, the column was washed with $300 \mathrm{~mL}$ washing solution and proteins were eluted with elution buffer $(1 \times$ PBS containing $100 \mathrm{mM}$ lactose (Sigma Aldrich), 0.02\% $\mathrm{NaN} 3,4 \mathrm{mM} \beta$-mercaptoethanol and $2 \mathrm{mM}$ EDTA). Fractions were analyzed for protein content using a NanoDrop ND-1000 (Isogen life science) after which positive samples were pooled and concentrated using Amicon 10K centrifugal filter units (Millipore). Protein was further purified using a Sephacryl S-100 sizing column (Sigma Aldrich), concentrated using Amicon $10 \mathrm{~K}$ centrifugal filter units and dialyzed against PBS using $7 \mathrm{~K}$ MWCO SnakeSkin (Thermo Fisher Scientific) containing $2 \mathrm{mM}$ $\beta$-mercaptoethanol at $4{ }^{\circ} \mathrm{C}$.

Alternatively, after concentration using Amicon $10 \mathrm{~K}$ centrifugal filter units, the protein solution was loaded onto a $5 \mathrm{~mL}$ GSTrap column (GE Healthcare) that was equilibrated with $25 \mathrm{~mL}$ binding buffer $(0.05 \mathrm{M}$ Tris- $\mathrm{HCl}, 0.5 \mathrm{M} \mathrm{NaCl}$, $\mathrm{pH}$ 7.4). The column was washed with $50 \mathrm{~mL}$ binding buffer and then incubated with $5 \mathrm{~mL}$ PBS containing 400 units Thrombin (GE Healthcare) for $16 \mathrm{~h}$ at $22^{\circ} \mathrm{C}$. Subsequently, a benzamidine HiTrap FF column (GE Healthcare) was washed with $1 \times$ PBS and attached to the GSTrap column. Columns were eluted with $10 \mathrm{~mL}$ elution buffer $(20 \mathrm{mM}$ Na2HPO4, $0.15 \mathrm{M} \mathrm{NaCl}, \mathrm{pH} 7.5$ ), and the eluate was dialyzed against $1 \times$ PBS containing $2 \mathrm{mM} \beta$-mercaptoethanol, and concentrated using Amicon $10 \mathrm{~K}$ centrifugal filter units.

As a final step, LPS was removed using Detoxi Gel (Thermo Fisher Scientific) according to the supplier's 
protocol. Protein concentration was determined using a BCA assay (Thermo Fisher Scientific), set to a stock of $1 \mathrm{mg} / \mathrm{mL}$ and aliquots were stored at $-80^{\circ} \mathrm{C}$ until further use.

\section{Hemagglutination assay}

In a 96-well round bottom plate (Greiner) $25 \mu \mathrm{L}$ of recombinant protein $(0.00-0.12-0.37-1.11-3.33 \mu \mathrm{M})$ in PBS was mixed with $25 \mu \mathrm{L}$ TSB buffer. Next, $25 \mu \mathrm{L}$ of $3 \%$ fixed rabbit red blood cells (Fitzgerald Industries International) in PBS was added and air bubbles were removed to prevent disturbance of the agglutination area. After 1-h incubation at room temperature, the plate was scanned and the agglutination diameter $(d)$ was measured using Adobe Photoshop CS4. The agglutination area $(A)$ was calculated by $A=\pi^{*}(d / 2)^{2}$. The relative agglutination area was calculated as: relative $A=A / A_{\text {blank }}^{-1}$.

\section{SDS-PAGE and Western Blotting}

Recombinant protein samples were subjected to sodium dodecyl sulfate polyacrylamide gel electrophoresis on a precast gradient polyacrylamide gel (2-20\%, BioRad). Following gel electrophoresis, the proteins were directly visualized using coomassie brilliant blue staining or proteins were transferred onto PVDF membranes (Millipore) following standard protocols. Subsequently, membranes were blocked with Rockland blocking buffer (Rockland Inc.) for $1 \mathrm{~h}$ at room temperature and incubated overnight at $4{ }^{\circ} \mathrm{C}$ with anti-galectin- 9 antibody (R\&D systems; $1: 250)$ in $0.5 \times \mathrm{PBS} / 0.1 \%$ Tween $/ 0.5 \times$ Rockland buffer. The membranes were washed using in $0.5 \times \mathrm{PBS} / 0.1 \%$ Tween $/ 0.5 \times$ Rockland buffer and incubated with a HRPlabeled rabbit anti-goat antibody (Dako; $1: 1000)$ for $1 \mathrm{~h}$ in $0.5 \times$ PBS $/ 0.1 \%$ Tween $/ 0.5 \times$ Rockland buffer. Finally, the membranes were washed with PBS/0.1\% Tween. Staining was visualized using the Pierce ECL Plus kit (Thermo Scientific), and images were obtained using a Uvitec imaging system (Uvitec).

\section{Proliferation assay}

The CellTiter-Glo assay (Promega) was used to assess the proliferation rate of endothelial cells. In short, 5000 HUVEC/well were seeded in $0.2 \%$ gelatin/PBS-coated 96-well plates. Cells were allowed to adhere for $2 \mathrm{~h}$ after which the culture medium was replaced. Cells were cultured in the presence or absence of recombinant galectin-9 proteins at the indicated concentrations for 3 days. Subsequently, the cells were washed with PBS to remove dead cells as well as cellular debris and incubated with $100 \mu \mathrm{L}$ CellTiter-Glo reagent for $10 \mathrm{~min}$ on a shaker at room temperature. Next, luminescence levels were determined using a microplate reader (Tecan). All experiments were performed in triplicate (three independent HUVEC sources) with three replicates per treatment condition within each experiment.

\section{Migration assay}

Endothelial cell migration was assessed by creating a scratch in a confluent layer of endothelial cells, followed by monitoring scratch closure in time in the presence or absence of recombinant galectin-9 proteins [23]. Images of each scratch were automatically acquired at $t=0$ and $t=7 \mathrm{~h}$ with a $1.4 \mathrm{Mb}$ GiGE color camera (Hitachi) on a DMI3000B microscope equipped with an automated xyz-stage (Leica) using Universal Grab software (version 6.3, DCllabs). Scratch area was determined using ImageJ. All experiments were performed in quadruplicate (three independent HUVEC sources) with three replicates per treatment condition within each experiment.

\section{Cell viability assay}

Endothelial cell viability was assessed by FACS following annexin V and 7AAD staining using the Annexin V Apoptosis Detection Kit (MabTag) according the manufacturers' protocol. In brief, 10.000 HUVEC were treated with $100 \mu \mathrm{M}$ of the different galectin- 9 isoforms for $8 \mathrm{~h}$. As positive control for induction of apoptosis, $20 \mu \mathrm{M}$ sunitinib was used. Following treatment, cells were harvested by trypsin digestion and stained while kept on ice. FACS analysis was performed on a FACSCalibur (BD Biosciences) according standard protocols. Experiments were performed on six different HUVEC isolates.

\section{Sprouting assay}

HUVEC cells were harvested and resuspended to a final concentration of $40 \mathrm{~K}$ cells $/ \mathrm{mL}$ in Methocel medium (RPMI containing 20\% Methocel and 10\% $0.22 \mu$ meter filtered heat-inactivated human serum). $25 \mu \mathrm{L}$ drops were pipetted onto the lid of a non-adhesive square Petri dish which was inverted and incubated overnight in a humidified incubator at $37{ }^{\circ} \mathrm{C}, 5 \% \mathrm{CO}_{2}$. The next day $200 \mu \mathrm{L}$ sprouting medium (10\% NBCS, $0.1 \%$ Heparin, $14.9 \%$ Methocel, $62.3 \%$ PureColl, $8.3 \% 10 \times 199$ complement, $4.5 \% \mathrm{NaOH}$ ) was added to each well of a $\mu$-Slide 8 well Ibidi plate (Ibidi) and left to solidify for $30 \mathrm{~min}$ at $37^{\circ} \mathrm{C}$. Meanwhile, the spheroids were harvested using a $1 \mathrm{~mL}$ pipet and pelleted. The pellet was carefully resuspended in $1.6 \mathrm{~mL}$ sprouting medium. 200 $\mu \mathrm{L}$ medium containing spheroids was added to each well and left to solidify for $30 \mathrm{~min}$ at $37^{\circ} \mathrm{C}$. Next, $100 \mu \mathrm{L}$ RPMI with or without $500 \mathrm{nM}$ recombinant galectin was applied topically and spheroids were allowed to sprout for $16 \mathrm{~h}$. Pictures of the spheroids were taken, and sprout length and number 
per spheroid were analyzed manually. All experiments were performed in triplicate, and per experiment at least 10 spheroids per condition were analyzed.

\section{Tube formation assay}

Tube formation was performed as described previously [23] with a few modifications. In brief, a flat-bottom 96-well plate was coated with $50 \mu \mathrm{L}$ Matrigel (BD Biosciences). Next, 20,000 HUVEC and 200 human perivascular cells (acquired from the vena saphena magna according to the Dutch guidelines for secondary use of material) were mixed and added to the wells. After $30 \mathrm{~min}$, medium with the appropriate concentration of compounds was added and cells were allowed to form tubular structures for $6 \mathrm{~h}$. Images were acquired with a $1.4 \mathrm{Mb}$ GiGE color camera (Hitachi) on a DMI3000B microscope equipped with an automated xyz-stage (Leica) using Universal Grab software (version 6.3, DCIlabs). Analyses of meshes were performed using ImageJ. Experiments were performed in quadruplicate.

\section{Chicken chorioallantoic membrane assay}

The chicken chorioallantoic membrane assay was performed as described before [24]. In brief, fertilized chicken eggs (Het Anker, the Netherlands) were incubated and rotated at a relative air humidity of $65 \%$ and a temperature of $37.6{ }^{\circ} \mathrm{C}$ in a hatching incubator with an automatic rotator for 3 days. On embryonic day 3 (EDD3), a small opening of approximately $3 \mathrm{~mm}$ in diameter was made in the eggshell at the top of the egg and sealed with tape. The eggs were then incubated for 3 days with the opening facing upwards. On EDD6, the eggs were opened further to allow easier access to the chorioallantoic membrane. For the topical application of recombinant galectins, polyethylene rings were deposited on the chorioallantoic membrane. From embryonic EDD6 to EDD9, recombinant galectins were applied daily within the polyethylene rings. Finally, on EDD10, pictures of the chorioallantoic membrane were taken using an Optech LFZ stereo microscope (Optech) after injection of approximately $1 \mathrm{~mL}$ contrast agent (zinc oxide in pure vegetable oil) under the chorioallantoic membrane and the ring. Quantification of different vascular parameters was obtained using HetCAM software (Peira). Experiments were performed in duplicate and at least 8 eggs per condition were used.

\section{Statistical analyses}

All data are presented as mean \pm SEM unless indicated otherwise. The Mann-Whitney rank sum test was used for comparison of the migration, proliferation and tube formation for which normal distribution could not be assumed. The Student's $t$ test was used for comparison of the CAM data, the apoptosis analyses, and the sprouting assay. $p$ values $<0.05$ were considered statistically significant, and all statistical analyses were performed using SPSS 20.0.0 (SPSS Inc.).

\section{Results}

We set out to determine the effect of the monovalent galectin-9 CRDs on endothelial cell function and angiogenesis. This was instigated by the observation that (i) the galectin-9 linker peptide is susceptible to proteolytic cleavage, (ii) both galectin-9 CRDs exert distinct activities on T-cell function, and (iii) endothelial cells express multiple galectin-9 mRNA splice variants, two of which lack the C-terminal CRD. Since galectin-9 expression was previously shown to also be induced by the inflammatory cytokine interferon gamma (IFNg) [25], we first determined the effect of this cytokine on galectin- 9 splice variant expression in endothelial cells. Following the treatment of HUVEC with IFNg for $24 \mathrm{~h}$, we observed a 10- to 20-fold induction in mRNA expression levels of all known galectin- 9 splice variants, including those lacking part of the coding sequence for the C-terminal CRD (Fig. 1a). To determine whether different microenvironments could differentially affect endothelial galectin-9 expression experiments were repeated using different cytokines and growth factors, including VEGF, TNF-alpha, IL-1 and IL-10 (Supplementary Fig. 1). Similar to INFg, IL-10 induced the expression of all galectin-9 isoforms albeit to a lesser extent. In contrast, both VEGF and IL-1 predominantly reduced the expression of most galectin-9 variants, especially of the dominant galectin-9M variant. TNF-alpha appeared to have only a limited effect on endothelial galectin-9 variant expression. These data suggest that the endothelial microenvironment controls endothelial galectin-9 splice variant expression.

To evaluate whether endothelial cell biology was differentially affected by the separate CRDs, we produced and isolated both domains as GST-coupled proteins as described previously [26]. Successful protein expression, GST removal and purification were confirmed by Western blot analysis (Fig. 1b). In addition, hemagglutination experiments were performed to assess the ability of the different isoforms to interact with and crosslink surface glycans. As expected, gal-9M showed a concentration-dependent increase in red blood cell agglutination. No agglutination was observed with either gal-9N, gal-9C or the combination of both (Fig. 1c).

Next, we compared the effect of both gal-9 CRDs with gal-9M on endothelial cell function in vitro. First, the effect on proliferation was determined in non-stimulated human umbilical vein endothelial cell (HUVEC) cultured under low serum conditions ( $2 \%$ human serum). In line with our previous observations, HUVEC proliferation was significantly hampered by $100 \mathrm{nM}$ gal-9M (Fig. 2a). This inhibitory effect 
Fig. 1 Expression, isolation and characterization of galectin- 9 isoforms. a Schematic representation of the different endothelial galectin-9 splice variants. Gal-9FL contains the full length linker sequence between the two CRDs. In gal-9M and gal-9S, the linker sequence is truncated by alternative splicing of exon 5 and exons 5/6, respectively. Further alternative splicing of exon 10 results in two additional isoforms, i.e., gal-9M $\Delta 10$ and gal9S $\Delta 10$. The bar graphs shows the fold difference in mRNA expression levels of these splice variants in HUVEC treated with interferon gamma compared to untreated cells. $n=3, * p<0.05$ versus control (dotted line). $\mathbf{b}$ Image of Western blot staining of recombinant galectin-9M (R\&D systems) and both galectin-9 CRDs (in house produced and purified). $\mathbf{c}$ Representative image of hemagglutination experiment with quantification of the agglutination area (bar graph on the right)

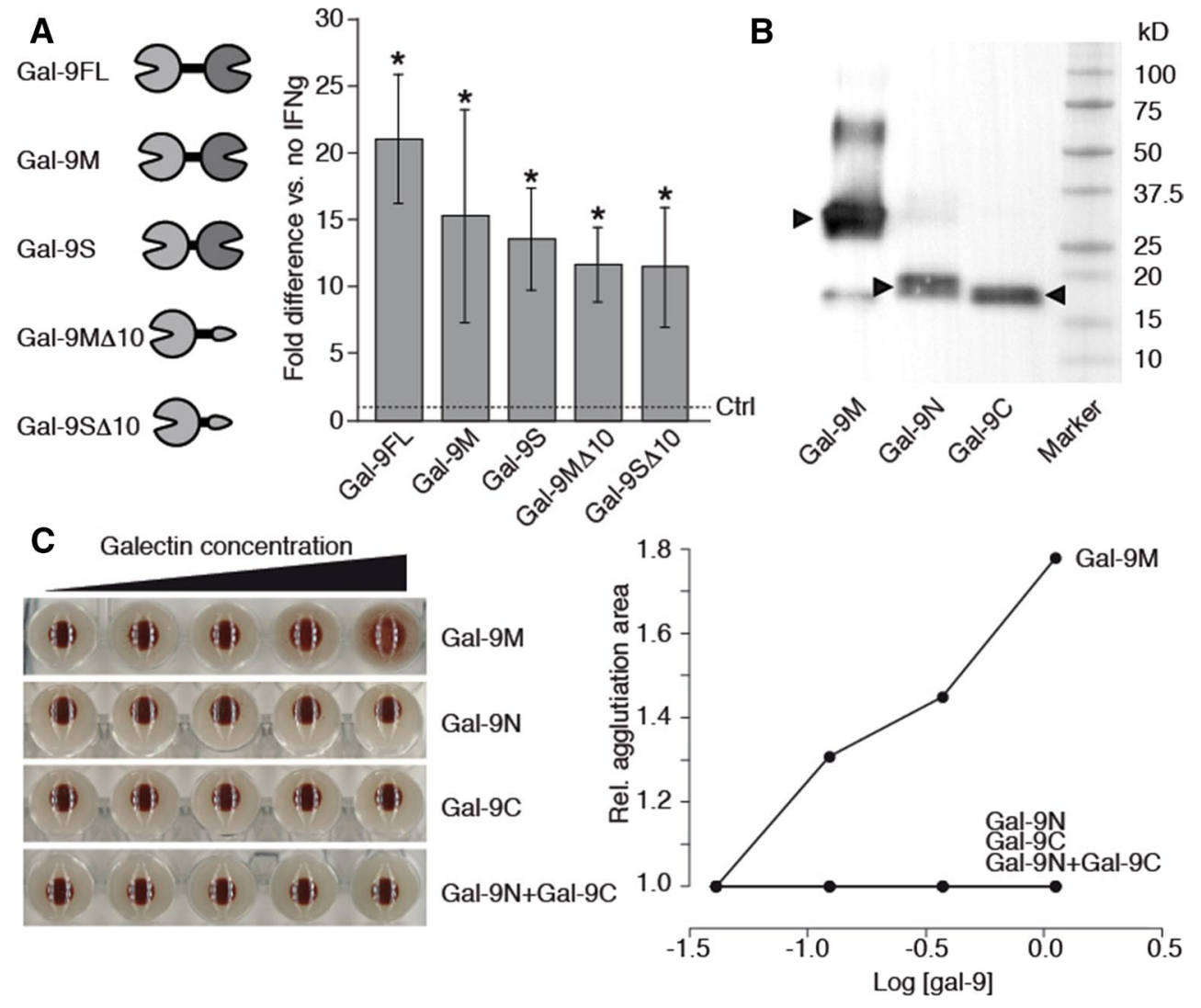

was not observed when HUVEC were cultured in the presence of either gal-9N or gal-9C. In fact, gal-9N and gal-9C appeared to slightly induce HUVEC proliferation (Fig. 2a). While gal-9M appeared to induce an opposite effect on proliferation as compared to gal-9N and gal-9C, the inhibitory effect of gal-9M was not neutralized by addition of either gal-9N or gal-9C (Supplementary Fig. 2a). However, addition of lactose neutralized the gal-9M effect indicating that this inhibition was carbohydrate-dependent. This was not observed when lactose was added to either gal-9N or gal-9C, suggesting that the effects of these isoforms were carbohydrate-independent (Supplementary Fig. 2b). Of note, the observed changes appeared not to be caused by altered cell survival since none of the galectin- 9 isoforms affected cell survival (Supplementary Fig. 3).

To evaluate whether the effects depended on endothelial activation status, similar experiments were performed with HUVEC cultured in the presence of $20 \%$ human serum. Of note, baseline proliferation of HUVEC in high-serum conditions was 1.6 -fold increased $(p<0.05)$ as compared to low serum conditions (data not shown). Treatment with gal-9M again appeared to inhibit proliferation although this did not reach statistical significance. Furthermore, there was no longer an effect of either gal-9N or gal-9C on HUVEC proliferation under high-serum conditions (Fig. 2b). These data suggest that the effect of different galectin-9 isoforms on EC proliferation depends on the cellular activation status. In addition, the individual CRDs show either no effect or an opposite effect on EC proliferation as compared to gal-9M.

Subsequently, we evaluated the effect of the different galectin isoforms on endothelial cell migration using the scratch wound assay. In line with previous observations, $100 \mathrm{nM}$ gal-9M did not affect migration in non-stimulated HUVEC. The same was observed for different concentrations of gal-9N and gal-9C (Fig. 2c). Interestingly, under high-serum conditions, i.e., stimulated HUVEC, gal-9M significantly induced migration while gal-9N had no effect and gal-9C inhibited migration (Fig. 2d). These data support the observation that either no or a reversed effect occurs when the separate gal-9 CRDs are compared to gal-9M. In addition, the effect of the different galectin isoforms appears to depend on the endothelial activation status.

We also assessed the effect of galectin-9 isoforms on sprout formation in a 3D collagen matrix. Dibenzazepine (DBZ), which was used as positive control, significantly induced HUVEC sprouting, as reflected by increased sprout numbers and sprout length (Fig. 3a). While we could not confirm the previously reported positive effect of gal-9M on endothelial sprouting, we did observe a significant inhibitory effect of gal-9C on both sprout number as well as sprout length (Fig. 3a). On the other hand, tube formation on a 

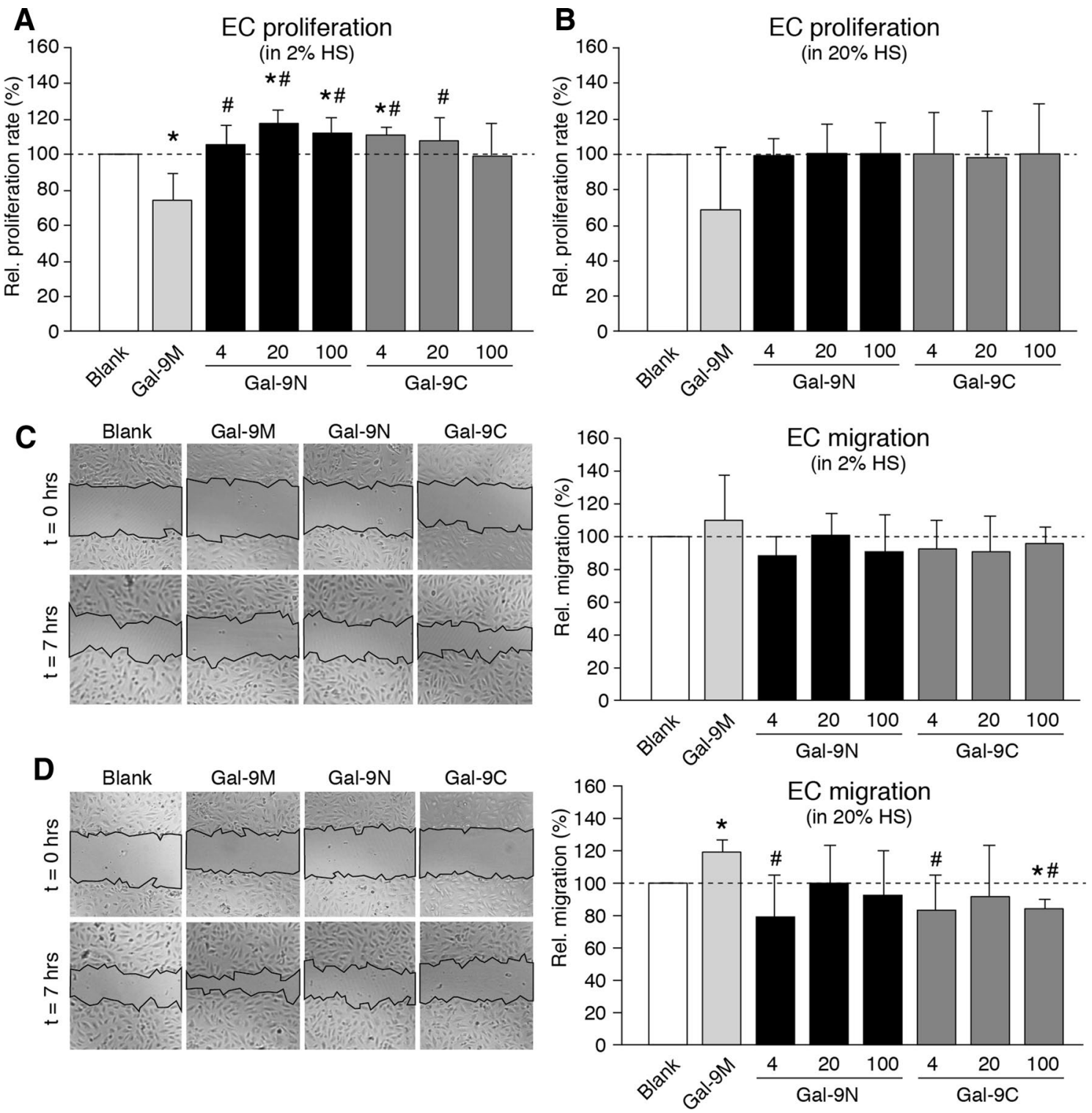

Fig. 2 Effect of galectin-9 isoforms on endothelial cell proliferation and migration. a-d The bar graphs show the relative proliferation $(\mathrm{A}+\mathrm{B})$ or migration $(\mathrm{C}+\mathrm{D})$ after treatment of quiescent $(2 \%$ HS; A + C) or activated (20\% HS; B + D) HUVEC with the different

galectin- 9 isoforms. The images in $\mathrm{C}+\mathrm{D}$ show representative pictures of the scratch area immediately after scratching $(t=0)$ and after $7 \mathrm{~h}$ of migration $(t=7)$. Proliferation, $n=3$; Migration, $n=4$. $^{*} p<0.05$ versus blank, $\# p<0.05$ versus gal-9M

collagen matrix appeared to be induced by all isoforms albeit only at low concentrations (Fig. 3b). The induction of tube formation was comparable to galectin-1.

Finally, the angioregulatory activity of the different isoforms in vivo was explored using the chicken chorioallantoic membrane (CAM) assay. For this, the CAM was locally treated with recombinant galectin- 9 proteins for 5 days after which images of the vasculature were taken and multiple vascular parameters were analyzed. From this, a general picture emerged indicating that all isoforms exert a moderate inhibitory effect on angiogenesis in vivo. While gal-9M only significantly reduced vessel length, both the separate CRDs induced a significant reduction in vessel length, total vessel area, and vessel branching (Fig. 4). The inhibitory effect of the gal-9N variant appeared to be the strongest but gal-9C was already inhibitory at lower concentrations. Collectively, our in vitro and in vivo studies revealed a complex functional activity of the different galectin-9 isoforms on endothelial cells and angiogenesis (summarized in Table 1). 
A

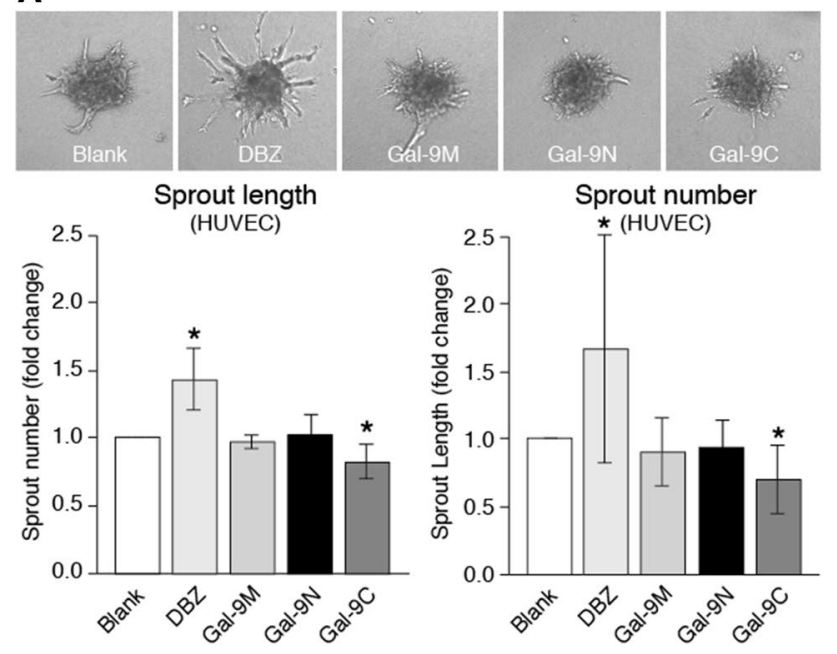

B
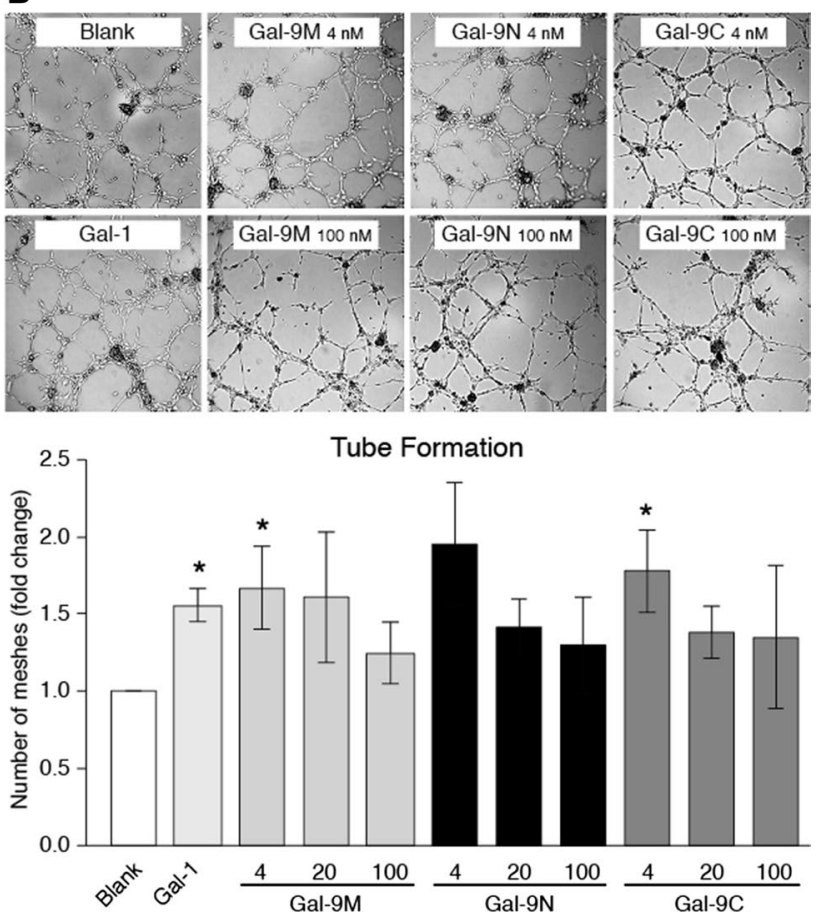

Fig. 3 Effect of galectin-9 isoforms on endothelial cell sprouting and tube formation. a The upper picture shows representative images of a HUVEC spheroid embedded in a collagen matrix for $16 \mathrm{~h}$. The bar graphs show the fold change in either sprout number (left panel) or sprout length (right panel) of treated spheroids relative to untreated spheroids (blank). Experiments were performed in triplicate and at least 10 spheroids were analyzed per experiment. $* p<0.05$ versus blank. b The upper picture shows representative images of HUVEC tube formation after $8 \mathrm{~h}$ on a collagen matrix. The bar graph shows the fold change in the number of meshes of treated cells relative to untreated cells (blank). Experiments were performed in triplicate. $* p<0.05$ versus blank

\section{Discussion}

In the current study, we explored the role of different galectin-9 isoforms in endothelial cell function and angiogenesis. Previous studies have shown that different galectin family members act as important regulators of blood vessel growth [4]. Others and we have shown that several galectins, including galectin-1, galectin-3, and galectin-8, can induce, facilitate and/or maintain endothelial cell function during angiogenesis [8, 10-12, 15, 27]. More recently, we reported on a potential role of galectin- 9 in endothelial cell biology [19]. Since alternative splicing [19] as well as proteolytic cleavage of the linker sequence [20] might result in the separation of both galectin-9 CRDs, the current study aimed to compare the angioregulatory activity of the dominant bivalent galectin-9 isoform (gal-9M) with the N-terminal galectin-9 CRD (Gal-9 N) and the C-terminal galectin-9 CRD (Gal-9C).

Our current data reveal a complex regulatory system by which the different galectin-9 isoforms affect endothelial cell function. For example, the bivalent gal-9M, appears to maintain the inactive state of quiescent endothelial cells by suppressing cell proliferation. However, both monovalent gal-9N and gal-9C isoforms slightly stimulate proliferation, suggesting that proteolytic cleavage by, e.g., thrombin, could reverse the activity of gal- 9 on endothelial cell proliferation. Indeed, interestingly, proteolytic cleavage of the $\mathrm{N}$-terminal fragment of galectin-3 has been shown to stimulate angiogenesis [13]. Whether this points toward a shared mechanism of proteolytic activation of galectins in angiogenesis needs further investigation.

In contrast to non-stimulated endothelial cells, the effects of the different isoform on proliferation were absent in already activated endothelial cells. It has previously been suggested that yet unknown serum components might neutralize the apoptotic effect of a recombinant galectin-9 isoform that lacks the endogenous linker region on melanoma cells [28]. We did not observe any effect of the different isoforms on endothelial cell apoptosis but we did observe that the different galectin- 9 isoforms induced significant effects on the migratory capacity of activated endothelial cells under high-serum conditions. Possibly, the serum components not only affect the ability of galectin-9 to hamper apoptosis in specific cells but also alter the activity of galectin- 9 in other cell-specific functions. Of note, the bivalent gal-9M displayed an opposite effect as compared to both CRDs, stimulation vs. inhibition, respectively. This further supports the idea that proteolytic cleavage can reverse or neutralize the activity of bivalent galectin-9. Collectively, our current data suggest that the angioregulatory activity of galectin- 9 depends on when and on how the different isoforms are presented to 

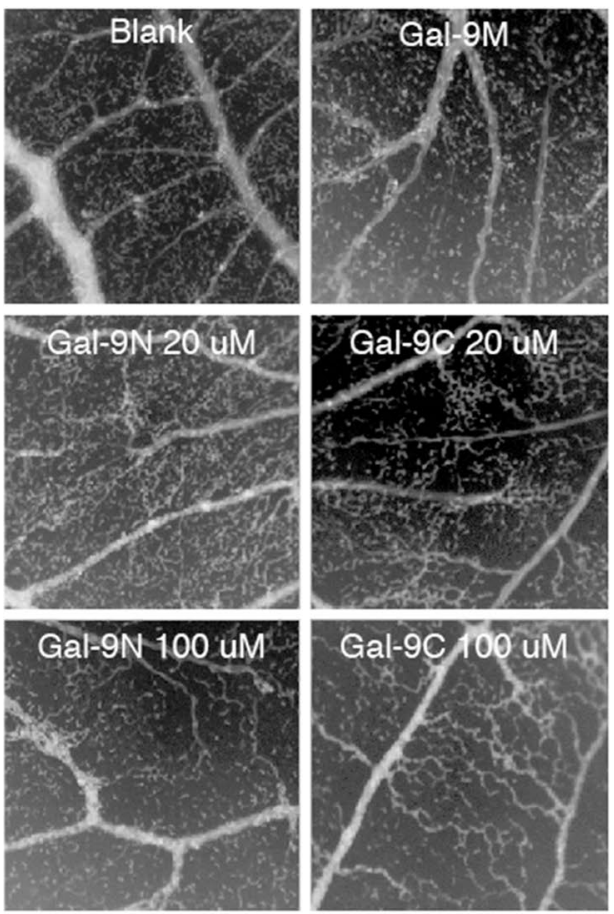
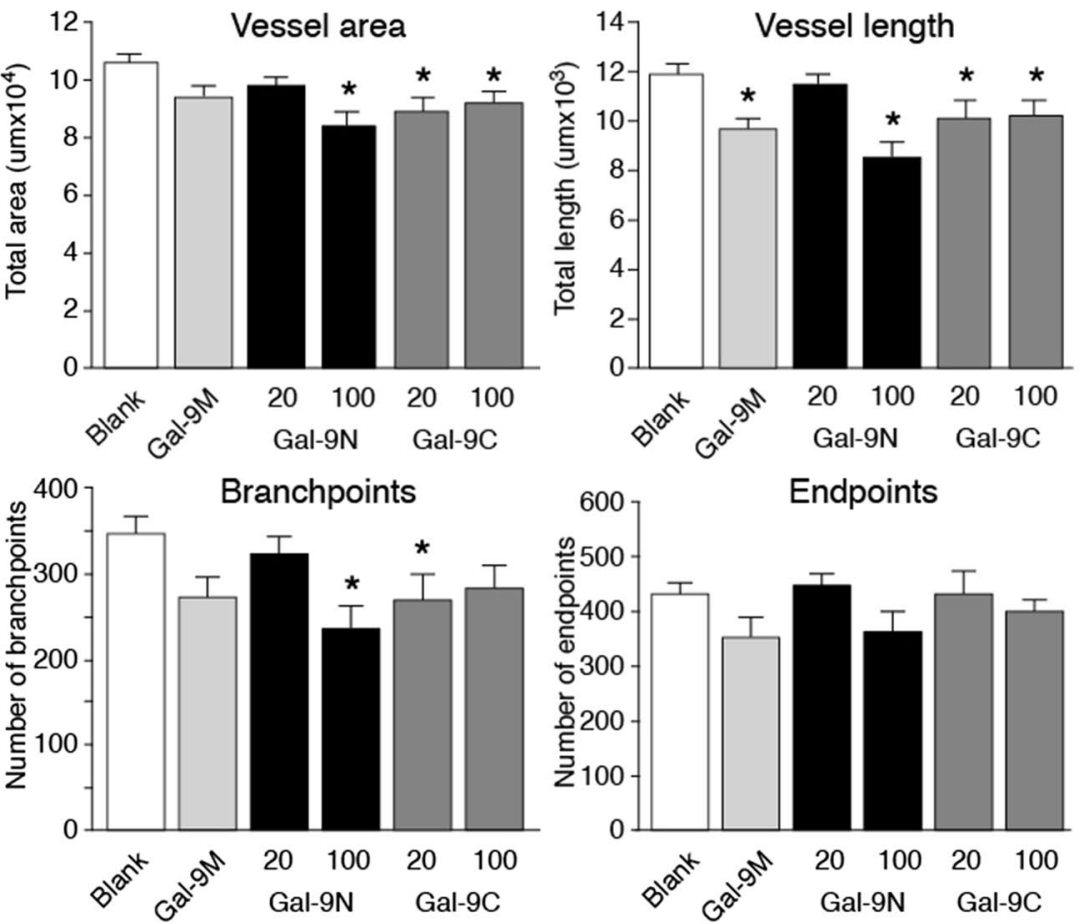

Fig. 4 Effect of galectin-9 isoforms on in vivo angiogenesis. The picture shows representative images of the CAM vasculature after 5 days of treatment with the different galectin-9 isoforms. The bar graphs show the quantification of different vascular parameters, i.e., total

vessel area, total vessel length, number of branch points and number of endpoints. CAM experiments were performed in duplicate with a total of 10 eggs per treatment in each experiment. $* p<0.05$ versus blank

Table 1 Effects of galectin-9 isoforms on endothelial cell function in vitro and angiogenesis in vivo

\begin{tabular}{|c|c|c|c|c|c|c|}
\hline & \multicolumn{2}{|l|}{ Gal-9M } & \multicolumn{2}{|l|}{ Gal-9N } & \multicolumn{2}{|l|}{ Gal-9C } \\
\hline & Quiescent & Activated & Quiescent & Activated & Quiescent & Activated \\
\hline Proliferation & - & $-1=$ & + & $=$ & + & $=$ \\
\hline Migration & $=$ & + & $=$ & $-1=$ & $=$ & $-1=$ \\
\hline Sprout formation & $=$ & & $=$ & & - & \\
\hline Tube formation & $+1=$ & & $+1=$ & & $+1=$ & \\
\hline CAM & $-1=$ & & $-1=$ & & $-1=$ & \\
\hline
\end{tabular}

- Inhibition, + stimulation, = no significant effect

or sensed by cells. This is supported by findings in other cell types. For example, galectin-9 is a potent inducer of T-cell apoptosis by binding to Tim-3 on these cells [29]. Nagahara et al. observed increased numbers of CD8+ T cells upon galectin-9 treatment in tumor bearing mice, while simultaneously CD4+ T-cell apoptosis was induced [30]. Gooden et al. also found that low-dose galectin-9 can activate and expand Th1 cells following an initial wave of T-cell apoptosis [31]. With regard to the specific CRDs, Bi et al. showed that the CRDs differentially regulate T-cell death induction [21]. The specificity of the C-terminal CRD was found to be the primary determinant of receptor recognition, T-cell death signaling, and susceptibility to cell death. In line with this, $\mathrm{Li}$ et al. showed a more potent role for Gal-9C in T-cell death compared to Gal-9N, while
Gal-9N was more effective in the activation of dendritic cells [32]. Moreover, Nishi et al. showed that thrombin mediated cleavage of the galectin-9 linker peptide reduced the chemoattraction of eosinophils [20]. All these data illustrate that both galectin-9 CRDs, either in the tandem repeat configuration or as single domains, display distinct activities on different cell types. This is further supported by our current findings in endothelial cells. Moreover, the distinct activity appears to depend not only on cell type or cell activation status but also on the galectin- 9 concentration. In fact, we occasionally observed a more potent activity at lower concentrations as compared to higher concentrations, e.g., tube formation and migration. This is in line with other studies showing biphasic activity of galectin-1 [11,31,33-35]. Of note, this complex role of 
galectin-9 in endothelial cell biology is a complicating factor when performing galectin-9 research. For example, while the majority of our current experiments confirmed our previous observations with galectin-9M [19], we could not confirm that galectin-9M induces sprouting when applied topically to the 3D matrix in which the endothelial spheroids are embedded. Most likely, this is due to a slight adaptation in the protocol (less Matrigel was used) that affected the galectin- 9 concentration gradient in the matrix. Thus, further studies are required to fully unravel how the cellular microenvironment influences galectin-9 isoform activity.

While the in vitro assays showed that the different galectin-9 isoforms exert different effects on endothelial cell function, the CAM assay results indicated that all isoforms hamper in vivo angiogenesis. Apparently, interfering with endothelial cell function by galectin-9, either by stimulation or inhibition, prevents the formation of an adequate and functional vasculature in vivo. Interestingly, of all the galectins that are expressed by endothelial cells, i.e., gal1/-3/-8/-9 [16], galectin-9 appears to be the only galectin with an inhibitory effect on angiogenesis. It remains to be established which mechanisms underlie this inhibitory effect. Thus far, the angiostimulatory activity of galectins has been linked to several signaling pathways, including the VEGF/VEGFR2 signaling axis, integrin signaling, and Ras signaling $[4,8,36]$. The ability of galectin-1, galectin-3 and galectin- 8 to trigger these signaling pathways has been linked to cross-linking of different receptors like VEGFR2, neuropilin-1, beta-integrins, and CD166 [4]. Whether galectin-9 also cross-links these receptors on endothelial cells remains to be established. In fact, most of the receptors found to interact with galectin-9 appear to be predominantly involved in immune cell activity and function, e.g., T-cell immunoglobulin mucin 3 (TIM-3), cell surface bound protein disulfide isomerases (PDI), CD40 and CD44. Interestingly, since several of these receptors are also expressed by endothelial cells it could be hypothesized that some are involved in regulating the effects of galectin-9 in angiogenesis. For example, it was suggested that galectin- 9 enhances T-cell migration by retaining PDI at the cell surfaces thereby facilitating CD61-mediated migration [37]. While speculative, a similar mechanism might be active in endothelial cells, especially since it has been shown that PDI can interact with integrins on endothelial cells [38]. Galectin-9 might also exert an angioregulatory role by interacting with CD44, a glycoprotein of which the expression was found to be induced upon endothelial cell activation [39]. Despite these potential candidates, future research should reveal which receptors are actually involved in mediating the galectin-9 effect on endothelial cells and to what extend receptor cross-linking is involved. Regarding the latter, it might be worthwhile to further explore the angioregulatory effects of the three bivalent galectin-9 isoforms, since the linker length influences the functional potency of galectins [18]. In addition, the susceptibility to proteolytic cleavage is affected by the linker length [20] which could be a mechanism to control how long cross-linked receptors can be retained on the cell surface.

Recently, Wiersma et al. have described that KRAS mutant colorectal cancer cells are more sensitive to galectin-9 induced cell death. This was linked to an elevated basal autophagy flux in the mutant cells that progressed into frustrated autophagy upon galectin-9 treatment [40]. While we did not evaluate autophagy, it will be of interest to explore to what extend galectin-9 interferes with these and other pathways. In addition, based on the opposing effects on migration and proliferation in quiescent and activated endothelial cells, it is tempting to speculate that the different galectin-9 isoforms are involved in controlling the switch between the tip cell and stalk cell phenotype during angiogenesis. Thus, exploring the role of galectin-9 in the delta-notch signaling pathway regulating this phenotype switch [41] is of interest for further studies.

It is important to note that the overall effects of the different galectin-9 isoforms on endothelial cell function in vitro and angiogenesis in vivo were subtle. Possibly, galectin-9 is mainly involved in fine-tuning the angiogenesis process. In that respect, it would be interesting to explore the effects of the galectin-9 isoforms in the presence of more potent angioregulatory galectins like, e.g., gal-1 and gal-3 as it has been shown that combining the latter two can potentiate the angiogenic activity of endothelial cells [42]. Whether this is true when galectin-9 is combined with other galectins requires further investigations.

In summary, we compared the angioregulatory activity of the bivalent galectin-9M protein with the two monovalent galectin-9 CRDs. We found distinct functional effects of the isoforms that appear to depend on endothelial cell activation status and galectin concentration. Our current data indicate that proteolytic processing of galectin-9 into two separate domains could serve as a negative feedback loop to neutralize or reverse the effect of the parent galectin-9M isoform. In addition, we observed that the galectin-9 isoforms hamper angiogenesis in vivo, which is different from other angioregulatory galectins. This could offer opportunities to exploit galectin-9 in pathologies that are associated with dysregulated angiogenesis, including cancer and cardiovascular diseases. All this warrants further investigation of the exact mechanisms by which galectin- 9 isoforms modulate endothelial cell function during angiogenesis.

Acknowledgements The authors thank Dr. Nozomu Nishi (Kagawa University, Japan) for providing the different galectin-9 constructs. This work was partly supported by a grant from the Dutch Cancer Society (VU2009-4358) to VLT. 
Open Access This article is distributed under the terms of the Creative Commons Attribution 4.0 International License (http://creativeco mmons.org/licenses/by/4.0/), which permits unrestricted use, distribution, and reproduction in any medium, provided you give appropriate credit to the original author(s) and the source, provide a link to the Creative Commons license, and indicate if changes were made.

\section{References}

1. Leffler H, Carlsson S, Hedlund M, Qian Y, Poirier F (2004) Introduction to galectins. Glycoconj J 19:433-440

2. Thijssen VL, Heusschen R, Caers J, Griffioen AW (2015) Galectin expression in cancer diagnosis and prognosis: a systematic review. Biochim Biophys Acta 1855:235-247

3. Liu FT, Rabinovich GA (2005) Galectins as modulators of tumour progression. Nat Rev Cancer 5:29-41

4. Thijssen VL, Rabinovich GA, Griffioen AW (2013) Vascular galectins: regulators of tumor progression and targets for cancer therapy. Cytokine Growth Factor Rev 24:547-558

5. Liu FT, Patterson RJ, Wang JL (2002) Intracellular functions of galectins. Biochim Biophys Acta 1572:263-273

6. Liu FT, Yang RY, Saegusa J, Chen HY, Hsu DK (2011) Galectins in regulation of apoptosis. Adv Exp Med Biol 705:431-442

7. Yang RY, Rabinovich GA, Liu FT (2008) Galectins: structure, function and therapeutic potential. Expert Rev Mol Med 10:e17

8. Croci DO, Cerliani JP, Dalotto-Moreno T, Méndez-Huergo SP, Mascanfroni ID, Dergan-Dylon S et al (2014) Glycosylationdependent lectin-receptor interactions preserve angiogenesis in anti-VEGF refractory tumors. Cell 156:744-758

9. Laderach DJ, Gentilini LD, Giribaldi L, Delgado VC, Nugnes L, Croci DO et al (2013) A unique galectin signature in human prostate cancer progression suggests galectin-1 as a key target for treatment of advanced disease. Cancer Res 73:86-96

10. Thijssen VL, Postel R, Brandwijk RJ, Dings RP, Nesmelova I, Satijn $S$ et al (2006) Galectin-1 is essential in tumor angiogenesis and is a target for antiangiogenesis therapy. Proc Natl Acad Sci USA 103:15975-15980

11. Thijssen VL, Barkan B, Shoji H, Aries IM, Mathieu V, Deltour L et al (2010) Tumor cells secrete galectin-1 to enhance endothelial cell activity. Cancer Res 70:6216-6224

12. Nangia-Makker P, Honjo Y, Sarvis R, Akahani S, Hogan V, Pienta KJ, Raz A (2000) Galectin-3 induces endothelial cell morphogenesis and angiogenesis. Am J Pathol 156:899-909

13. Nangia-Makker P, Wang Y, Raz T, Tait L, Balan V, Hogan V, Raz A (2010) Cleavage of galectin-3 by matrix metalloproteases induces angiogenesis in breast cancer. Int J Cancer 127:2530-2541

14. Markowska AI, Liu FT, Panjwani N (2010) Galectin-3 is an important mediator of VEGF- and bFGF-mediated angiogenic response. J Exp Med 207:1981-1993

15. Cardenas Delgado VM, Nugnes LG, Colombo LL, Troncoso MF, Fernandez MM, Malchiodi EL et al (2011) Modulation of endothelial cell migration and angiogenesis: a novel function for the "tandem-repeat" lectin galectin-8. FASEB J 25:242-254

16. Thijssen VL, Hulsmans S, Griffioen AW (2008) The galectin profile of the endothelium: altered expression and localization in activated and tumor endothelial cells. Am J Pathol 172:545-553

17. Heusschen R, Griffioen AW, Thijssen VL (2013) Galectin-9 in tumor biology: a jack of multiple trades. Biochim Biophys Acta 1836:177-185

18. Earl LA, Bi S, Baum LG (2011) Galectin multimerization and lattice formation are regulated by linker region structure. Glycobiology 21:6-12

19. Heusschen R, Schulkens IA, van Beijnum J, Griffioen AW, Thijssen VL (2014) Endothelial LGALS9 splice variant expression in endothelial cell biology and angiogenesis. Biochim Biophys Acta 1842:284-292

20. Nishi N, Itoh A, Shoji H, Miyanaka H, Nakamura T (2006) Galectin- 8 and galectin-9 are novel substrates for thrombin. Glycobiology $16: 15 \mathrm{C}-20 \mathrm{C}$

21. Bi S, Earl LA, Jacobs L, Baum LG (2008) Structural features of galectin-9 and galectin-1 that determine distinct $\mathrm{T}$ cell death pathways. J Biol Chem 283:12248-12258

22. Levi G, Teichberg VI (1981) Isolation and physicochemical characterization of electro lectin, a beta-D-galactoside binding lectin from the electric organ of Electrophorus electricus. J Biol Chem 256:5735-5740

23. Schulkens IA, Kleibeuker EA, Castricum KC, Griffioen AW, Thijssen VL (2015) Examination of the role of galectins and galectin inhibitors in endothelial cell biology. Methods Mol Biol 1207:285-291

24. Kleibeuker EA, Schulkens IA, Castricum KC, Griffioen AW, Thijssen VL (2015) Examination of the role of galectins during in vivo angiogenesis using the chick chorioallantoic membrane assay. Methods Mol Biol 1207:305-315

25. Imaizumi T, Kumagai M, Sasaki N, Kurotaki H, Mori F, Seki $M$ et al (2002) Interferon- $\{$ gamma $\}$ stimulates the expression of galectin-9 in cultured human endothelial cells. J Leukoc Biol 72:486-491

26. Nishi N, Itoh A, Fujiyama A, Yoshida N, Araya S, Hirashima M et al (2005) Development of highly stable galectins: truncation of the linker peptide confers protease-resistance on tandemrepeat type galectins. FEBS Lett 579:2058-2064

27. Freitag N, Tirado-González I, Barrientos G, Herse F, Thijssen VL, Weedon-Fekjær SM et al (2013) Interfering with Gal1 -mediated angiogenesis contributes to the pathogenesis of preeclampsia. Proc Natl Acad Sci USA 110:11451-11456

28. Wiersma VR, de Bruyn M, van Ginkel RJ, Sigar E, Hirashima M, Niki T et al (2012) The glycan-binding protein galectin-9 has direct apoptotic activity toward melanoma cells. J Invest Dermatol 132:2302-2305

29. Zhu C, Anderson AC, Schubart A, Xiong H, Imitola J, Khoury SJ et al (2005) The Tim-3 ligand galectin-9 negatively regulates T helper type 1 immunity. Nat Immunol 6:1245-1252

30. Nagahara K, Arikawa T, Oomizu S, Kontani K, Nobumoto A, Tateno $\mathrm{H}$ et al (2008) Galectin-9 increases Tim-3+dendritic cells and CD8+ T cells and enhances antitumor immunity via galectin-9-Tim-3 interactions. J Immunol 181:7660-7669

31. Gooden MJ, Wiersma VR, Samplonius DF, Gerssen J, van Ginkel RJ, Nijman HW et al (2013) Galectin-9 activates and expands human T-helper 1 cells. PLoS ONE 8:e65616

32. Li Y, Feng J, Geng S, Geng S, Wei H, Chen G et al (2011) The $\mathrm{N}$ - and C-terminal carbohydrate recognition domains of galectin-9 contribute differently to its multiple functions in innate immunity and adaptive immunity. Mol Immunol 48:670-677

33. Adams L, Scott GK, Weinberg CS (1996) Biphasic modulation of cell growth by recombinant human galectin-1. Biochim Biophys Acta 1312:137-144

34. Sakaguchi M, Shingo T, Shimazaki T, Okano HJ, Shiwa M, Ishibashi $S$ et al (2006) A carbohydrate-binding protein, Galectin-1, promotes proliferation of adult neural stem cells. Proc Natl Acad Sci USA 103:7112-7117

35. Vas V, Fajka-Boja R, Ion G, Dudics V, Monostori E, Uher F (2005) Biphasic effect of recombinant galectin-1 on the growth and death of early hematopoietic cells. Stem Cells 23:279-287

36. Blaževitš O, Mideksa YG, Šolman M, Ligabue A, Ariotti N, Nakhaeizadeh H et al (2016) Galectin-1 dimers can scaffold Raf-effectors to increase H-ras nanoclustering. Sci Rep 6:24165

37. Bi S, Hong PW, Lee B, Baum LG (2011) Galectin-9 binding to cell surface protein disulfide isomerase regulates the redox 
environment to enhance T-cell migration and HIV entry. Proc Natl Acad Sci 108:10650-10655

38. Swiatkowska M, Szymański J, Padula G, Cierniewski CS (2008) Interaction and functional association of protein disulfide isomerase with alphaVbeta3 integrin on endothelial cells. FEBS J 275:1813-1823

39. Griffioen AW, Coenen MJ, Damen CA, Hellwig SM, van Weering DH, Vooys W et al (1997) CD44 is involved in tumor angiogenesis; an activation antigen on human endothelial cells. Blood 90:1150-1159

40. Wiersma VR, de Bruyn M, Wei Y, van Ginkel RJ, Hirashima M, Niki $T$ et al (2015) The epithelial polarity regulator LGALS9/ galectin-9 induces fatal frustrated autophagy in KRAS mutant colon carcinoma that depends on elevated basal autophagic flux. Autophagy 11:1373-1388
41. Blanco R, Gerhardt H (2013) VEGF and Notch in tip and stalk cell selection. Cold Spring Harb Perspect Med 3:a006569

42. D'Haene N, Sauvage S, Maris C, Adanja I, Le Mercier M, Decaestecker $\mathrm{C}$ et al (2013) VEGFR1 and VEGFR2 involvement in extracellular galectin-1- and galectin-3-induced angiogenesis. PLoS ONE 8:e67029 